\title{
AMBICIONES, CONTRADICCIONES $Y$ PERSPECTIVAS DE LA POLÍTICA COMERCIAL DE LA UNIÓN EUROPEA
}

\begin{abstract}
Resumen
Como pilar fundamental de la política comercial comunitaria, la Unión Europea (UE) está realizando un enorme esfuerzo institucional $y$ negociador para el establecimiento de acuerdos comerciales con terceros países. Estos Acuerdos, forman parte de una estrategia más amplia en la búsqueda de mayor competitividad de Europa, mediante el acceso a nuevos mercados para el comercio y la inversión. Como objetivos fundamentales de los Acuerdos, negociados de forma creciente con agrupaciones regionales (MERCOSUR, Comunidad Andina, CARIFORUM, ASEAN, SADC en África, entre otros) se incluyen, además, los de la lucha contra la pobreza, el logro de la cohesión social, o el fomento de la integración regional y la democracia. Algunos de estos objetivos, cimentados en valores que trascienden de la esfera estrictamente económica, son la base del nuevo modelo de relaciones comerciales exteriores de la UE, que se contrapone en la retórica europea, a otras formas de relación por otros actores internacionales, como Estados Unidos o China.
\end{abstract}

\section{Palabras clave}

Política comercial, acuerdos comerciales, desarrollo.
Por: Ainhoa Marín Egoscozábal*

\section{INTRODUCCIÓN}

\begin{abstract}
Uas relaciones económicas externas de la Unión Europea (UE) se fundamentan en tres grandes ámbitos: la política exterior comunitaria, las relaciones comerciales con el exterior y la política de cooperación para el desarrollo. De los tres ámbitos señalados $y$, de entre las políticas relacionadas con los mismos, la política comercial tiene la peculiaridad de ser la única común en sentido estricto. Es decir, es competencia exclusiva de las instituciones comunitarias. No ocurre así en el caso de las políticas no comunes, donde las líneas de actuación de la UE se añaden a las de cada Estado miembro1.
\end{abstract}

Las políticas sobre las que se construyen las relaciones exteriores de la UE no siempre han

\footnotetext{
* Doctora en CC.Económicas y Empresariales por la UNED. Profesora de Economía Aplicada de la Universidad de Nebrija.

1 Así, por ejemplo, la política de cooperación de la UE es la suma de la cooperación que realiza la Comisión Europea, más las políticas de cooperación de cada Estado miembro.
} 
perseguido objetivos coincidentes. La UE ha emprendido un renovado esfuerzo de coherencia de objetivos, sobre todo desde la Cumbre de Monterrey de 2002, donde se estableció como compromiso comunitario, que los objetivos de desarrollo debían estar presentes y permear en todas las políticas comunitarias que tuvieran implicaciones sobre los países en desarrollo.

Paralelamente, la revisión de la Estrategia de Lisboa, realizada en el año 2005, incorporó la necesidad de disponer de mercados abiertos en el exterior, fuera de las fronteras de la UE, como una de las acciones clave para el logro de los objetivos de Lisboa ${ }^{2}$. La política comercial adquiere desde ese momento un papel instrumental fundamental para lograr el crecimiento económico y contribuir a una mayor competitividad de Europa. La firma de acuerdos comerciales con países terceros, se convierte así en parte esencial de la política comercial comunitaria, como instrumento facilitador de la eliminación de obstáculos al comercio en los mercados internacionales, a través de la puesta en marcha de relaciones especiales con algunos países. La Comisión Europea, que es la institución que tiene conferido el mandato de negociar los diferentes acuerdos internacionales, haido configurando progresivamente una importante red de relaciones comerciales a escala mundial, tal y como se comentará posteriormente.

En definitiva, la firma de acuerdos con países terceros es el paradigma comunitario donde deberían confluir los objetivos de las diferentes políticas comunitarias, en aras de la deseable coherencia de políticas. Esencialmente, los acuerdos deberían servir de apoyo a la mejora

\footnotetext{
2 La Estrategia de Lisboa de 2000, revisada posteriormente en el 2005, tenía como objetivo alinear las políticas e instrumentos comunitarios con el fin de mejorar la competitividad de la UE, acelerar el crecimiento económico y crear puestos de trabajo. Todo ello, sobre la base de un modelo de garantías sociales para los/las ciudadanos/as de la UE.
}

\section{A bstract}

Considered as a key issue in the European Union's Trade Policy, the EU is committing strong institutional efforts and time-consuming negotiations for the establishment of trade agreements with third countries. These agreements are part of a broader strategy for enhancing competitiveness in Europe, through broader and bigger markets for trade and investment. The agreements are commonly negotiated with regional groupings (MERCOSUR, Andean Community, CARIFORUM, ASEAN, $S A D C$ in Africa, amongst others) and aim at eradicating poverty, achieving social cohesion, or enhancing regional integration and democracy. Some of these goals are values that go far beyond economic issues, and configure the renewed model of external EU relations, often opposed to the USA or Chinese relations with third countries.

\section{KEY WORDS}

Commercial policy- commercial agreements- development. 
de la competitividad de Europa, para de esta manera, lograr mercados abiertos que permitan la entrada de empresas europeas y unas más fluidas relaciones comerciales; pero igualmente, cuando los acuerdos impliquen a países en desarrollo, deberían propender por la lucha contra la pobreza y otras ambiciones que entran dentro del ámbito del desarrollo humano. La UE está planteando así un modelo diferente de relaciones con países terceros, que pretende oponer al modelo norteamericano y de otras potencias (como por ejemplo, China), donde el objetivo de desarrollo humano aparece de forma secundaria o es inexistente y donde se resaltan los beneficios macroeconómicos como prioritarios ${ }^{3}$.
La UE está así en proceso de negociación o tiene establecidos, bajo la denominación de Acuerdos de Asociación Económica, acuerdos ya firmados (por ejemplo, con Chile y Mercosur) o en proceso de negociación (Mercosur, Comunidad Andina, África Subsahariana, Caribe y Pacífico entre otros). Estos Acuerdos se fundamentan en tres pilares de relación: diálogo político, cooperación y comercio. El logro de Acuerdos que sirvan de instrumento eficiente para la apertura de mercados y mejora de la competitividad de los europeos y además sean coherentes con los objetivos de desarrollo humano de los países, es probablemente uno de los retos más importantes a los que se enfrentan las relaciones exteriores de la UE en este milenio.

\section{LA POLIÍTICA COMERCIAL COMUNITARIA: RASGOS GENERALES}

\begin{abstract}
Ta política comercial común puede definirse como el conjunto de competencias de la Unión Europea en el ámbito de las relaciones comerciales con terceros países y constituye uno de los principales instrumentos de las relaciones exteriores de la Unión Europea. Esta es una de las cuatro políticas comunes previstas en el Tratado de Roma, junto a la política agrícola comunitaria (PAC), la política de competencia y la política de transportes e implica la gestión uniforme de las relaciones comerciales con los terceros países.
\end{abstract}

La política comercial de la UE es consecuencia de la implantación de la unión aduanera. Recuérdese que el Tratado de Roma de 1957 estableció como objetivo crear entre los Estados miembros una unión aduanera donde los intercambios estarían libres de todo obstáculo y se aplicaría un arancel exterior común a las importaciones procedentes de terceros Estados. La política comercial común se refiere principalmente a los intercambios de mercancías; sin embargo, desde la nueva redacción del art. 133 aprobada en Niza, se refiere, además, al comercio de servicios y a los aspectos comerciales de la propiedad intelectual. De las cuatro modalidades de prestación de servicios, sólo para suministro transfronterizo de servicios, la Comisión posee competencia exclusiva. Para el resto de modalidades (consumo en el extranjero, presencia comercial y presencia de personas

\footnotetext{
2 En este sentido, las crecientes inversiones del Gobierno de China en algunos países africanos, sobre todo en el sector energético, están siendo profusamente criticadas en el entorno europeo, por no tener en cuenta las limitaciones democráticas en los mismos, o los derechos humanos de la población. Es, además frecuente, el desplazamiento de mano de obra china y el condicionamiento de las inversiones al contrato de suministros originarios de China, con lo que las voces más críticas señalan los escasos beneficios para la población africana desde la perspectiva del desarrollo.
} 
físicas) se comparte la competencia con los Estados miembros. En cuanto a los derechos de propiedad intelectual relacionados con el comercio (ADPIC), la Comunidad y los Estados miembros tienen por lo general, competencias compartidas.

La Unión Europea es, en la actualidad, el primer protagonista mundial del comercio internacional, por delante de Estados Unidos y Japón. En un contexto de fuerte crecimiento de los intercambios internacionales, la política comercial común se ha configurado así como una de las políticas más importantes de la Comunidad. La política comercial se apoya en una serie de instrumentos, de carácter interno y externo, con los que la UE regula las relaciones comerciales en los ámbitos intracomunitario y extracomunitario.

A continuación nos referiremos a los instrumentos que corresponden a la faceta externa de la política comercial de la UE.

\section{LOS INSTRUMENTOS DE LA POLÍTICA COMERCIAL COMUNITARIA}

En el marco de la política comercial europea y, en su dimensión de relaciones comerciales con países terceros, se han desarrollado los siguientes instrumentos: el arancel exterior común (AEC); los instrumentos de defensa comercial, los instrumentos de acceso a los mercados de los terceros países y los acuerdos comerciales con terceros países.

\subsection{El Arancel Exterior Común (AEC)}

El arancel exterior común (AEC), característica esencial de la unión aduanera europea, consiste en la aplicación de derechos de aduana uniformes a los productos importados de terceros países, independientemente de cuál sea el Estado miembro de destino. Supone por tanto, la homogeneización de la protección de los Estados miembros frente a países no comunitarios en el ámbito del comercio. En su origen, fue calculado con la media aritmética de los aranceles aduaneros aplicados en 1957 por los Estados fundadores, aunque ha sido desde entonces modificado en numerosas ocasiones por el Consejo.

\subsection{Los instrumentos de defensa comercial}

La Unión Europea, como la mayoría de los países, dispone de un sistema de instrumentos de defensa comercial que tiene por objeto defender a sus productores contra las importaciones consideradas desleales o subvencionadas y protegerse de cambios importantes de los flujos comerciales que sean nocivos para la economía comunitaria. Los instrumentos de defensa comercial son de tres tipos: las medidas «antidumping», medidas antisubvenciones y medidas de salvaguardia. La UE utiliza estas medidas sobre la base de las normas acordadas en el marco de la Organización Mundial del Comercio (Comisión Europea, 2006a): 
Las medidas «antidumping» son el instrumento de defensa ante la importación de bienes procedentes de terceros países a precios inferiores a los que tienen en el mercado interior productos similares. Estas, que se supone, son el instrumento de defensa más utilizado de la UE, permiten adoptar medidas correctoras para eliminar el perjuicio (generalmente, se impone un derecho a las importaciones). Por otro lado, las medidas antisubvenciones se utilizan para impedir la importación de productos mantenidos artificialmente a bajo precio, mediante subvenciones públicas en el tercer país de origen, si se demuestra que tales subvenciones pueden perjudicar a los productores de la UE. Al igual que las medidas «antidumping», permiten el restablecimiento temporal de derechos de aduana sobre las importaciones en cuestión.

Las medidas de salvaguardia, como tercer instrumento de defensa comercial, permiten a la UE imponer restricciones de corta duración a la importación de productos, siempre que se pueda probar que importaciones que se pretenden reducir han aumentado rápidamente y en masa, causando así un perjuicio a los productores nacionales. Estas medidas deben ser no discriminatorias; es decir, deben aplicarse a todas las importaciones del producto contemplado sin distinción de origen.
Así, la UE mantiene diferentes mecanismos de salvaguardia, que previenen las importaciones que aumentan en tales cantidades y, por lo tanto, pueden representar un riesgo de perjuicio grave para la industria europea. Estos instrumentos, que pueden servir de referencia, han permitido poner bajo vigilancia diferentes productos textiles y de calzado de China y han funcionado para la defensa europea de cítricos procedentes de China, o de salmón de Noruega, siempre de forma temporal.

\subsection{Los instrumentos de acceso a los mercados exteriores}

la Unión Europea se ha dotado de mecanismos que tienen como finalidad detectar los obstáculos al comercio con que se encuentran las empresas comunitarias en sus exportaciones hacia terceros países. En este sentido, se ha articulado un procedimiento que permite a las empresas pedir a las instituciones comunitarias que reaccionen frente a obstáculos detectados en la exportación de productos a terceros países. La UE considera como obstáculo al comercio, toda práctica comercial adoptada por un tercer país, pero prohibida por las normas del comercio internacional, que reconocen a la parte perjudicada el derecho a tratar de eliminar su efecto. Las normas comerciales internacionales son las negociadas en el marco de la OMC o las derivadas de acuerdos bilaterales entre la UE y el país en cuestión. 


\section{LOS ACUERDOS COMERCIALES DE LA UE CON TERCEROS PAÍSES}

I a Comisión Europea ha negociado diferentes acuerdos internacionales y ha ido configurando progresivamente una importante red de relaciones comerciales a escala mundial. Estos acuerdos suponen un instrumento fundamental de la política comercial europea, a través de los cuales se pretende conseguir la eliminación de obstáculos al comercio en los mercados internacionales, mediante la puesta en marcha de relaciones especiales con algunos países. Los acuerdos entre la UE y terceros países no se ajustan a un único modelo. En la práctica, el establecimiento de acuerdos comerciales supone, generalmente, otorgar un tratamiento comercial más favorable al régimen general, en el marco de la creación de áreas de libre comercio o uniones aduaneras, por ejemplo. Una zona de libre comercio supone la eliminación de las restricciones comerciales, entre los países, pero cada uno mantiene en vigor frente al exterior su propio arancel aduanero y su política comercial. Las uniones aduaneras implican el compromiso para la libre circulación entre los países que la conforman y, además, se aplica un arancel aduanero y una política comercial común frente a las importaciones de terceros países.

En las relaciones con los países en desarrollo, la UE presenta dos grandes líneas de actuación: o bien se han establecido disposiciones comerciales no recíprocas (por las que sólo el mercado de la UE se liberaliza para la entrada de productos procedentes de los países en desarrollo) o se establecen acuerdos recíprocos (basados en la liberalización mutua) que adoptan generalmente la forma de acuerdos de libre comercio. En el primer caso, encontramos, por ejemplo, un tratamiento comercial preferencial para los países en desarrollo, en el marco del Sistema de Preferencias Generalizadas (SPG) 4 .

En el caso de acuerdos recíprocos de la UE con terceros países, estos están tendiendo a incluir de forma creciente, aspectos comerciales adicionales a los aranceles (comercio de servicios, inversión, temas de propiedad intelectual, entre otros, tal y como se comenta más adelante), así como aspectos relacionados con programas de cooperación y temas políticos. Por ello, la propia UE se refiere a ellos como «acuerdos globales», o de nueva generación.

Desde una perspectiva geográfica, los principales acuerdos que la UE ha celebrado con terceros países son:

4 Por el Sistema de Preferencias Generalizadas (SPG), la UE otorga un acceso con aranceles más bajos al mercado comunitario, a las importaciones procedentes de 178 países y territorios en desarrollo. El esquema revisado del SGP, en vigor desde enero de 2006, comprende tres tipos de regímenes: el régimen general (y menos favorable en términos de concesiones comerciales), el SPG+ (que se comenta más adelante) y, finalmente, la iniciativa «Todo menos armas» de 2000, por la cual la UE concede acceso libre de derechos y de contingentes a los productos procedentes de los denominados «países menos adelantados» (según la lista de Naciones Unidas), dentro del grupo de países en desarrollo. La diferencia fundamental entre el SPG, en cualquiera de sus tres modalidades y un acuerdo comercial bilateral está en la no reciprocidad. Bajo el SPG, los países en desarrollo que reciben las concesiones comerciales no tienen la obligación de otorgar, a su vez, preferencias comerciales a la entrada de productos europeos en sus mercados. 
- El Espacio Económico Europeo (EEE).

- Acuerdos con los países de África, Caribe y Pacífico (ACP).

- Acuerdos Mediterráneos y con países de Oriente Medio.

- Acuerdos con países de América Latina.

\subsection{El Espacio Económico Europeo (EEE)}

El Espacio Económico Europeo (EEE) es una zona de libre comercio o área de libre cambio, integrada por los miembros de la UE, junto con Noruega, Islandia y Liechtenstein. El Acuerdo EEE se firmó en mayo de 1993 y consiste básicamente en el establecimiento de las denominadas «cuatro libertades» del mercado único, entre los países que lo conforman; es decir: la libre circulación de mercancías, de servicios, de capitales y, finalmente, la libre circulación de personas.

A pesar de estar basado en estas cuatro libertades, junto con algunas políticas de acompañamiento (política de competencia, la protección del consumidor o del medio ambiente, entre otras), el EEE no constituye una unión aduanera, pues no hay una política común de protección comercial frente al exterior. Además, excluye los productos agrícolas y de pesca, con lo que no supone un mercado para el comercio libre de barreras de forma completa. Conviene resaltar, por otra parte, que aunque no forman parte del EEE, en el ámbito continental la UE tiene también establecidos acuerdos de libre comercio con Suiza y ha celebrado acuerdos de unión aduanera con Andorra y San Marino.

\subsection{Los Acuerdos de Asociación con África, Caribe y Pacífico (ACP)}

Desde 1975, los Acuerdos o Convenciones de Lomé han constituido el marco de relación entre la Unión Europea y el denominado grupo África, Caribe y Pacífico (ACP). En virtud de los sucesivos Acuerdos de Lomé, los productos procedentes de 78 países de África Subsahariana, Caribe y Pacífico han recibido un acceso libre de aranceles para todos los productos industriales y la mayoría de los agrícolas. El esquema Lomé se ha considerado durante décadas un modelo para la cooperación con los países en desarrollo, que completaba el apartado comercial con asistencia financiera y técnica para el desarrollo.

Desde la expiración del cuarto Convenio Lomé, la UE ha trazado una nueva estrategia comercial y de desarrollo con los países ACP con base en elAcuerdo de Cotonú de 2000. Entre los contenidos comerciales del Acuerdo de Cotonú, se recoge el mandato de negociar, con fecha límite de 2008; Acuerdos de Asociación Económica («Economic Partnership Agreements» EPAs) entre la UE y grupos de países del grupo ACP. Los EPAs suponen el establecimiento de áreas de libre comercio, acompañados de asistencia técnica y financiera para el desarrollo, con el objetivo fundamental de reducir la pobreza en los países ACP.

Durante el proceso de negociación de los EPAs, en marcha con seis agrupaciones regionales en el seno del Grupo ACP (cuatro africanas, una en Caribe y otra que aglutina a los Estados del Pacífico), la Comisión Europea está introduciendo temas relacionados con el comercio, más allá de los estrictamente relacionados con la liberalización arancelaria. Así, es probable que aparezcan los textos de los futuros Acuerdos con temas como la facilitación del comercio (simplificación de 
trámites y reglamentación aduanera); normas para facilitar la inversión extranjera, normativa referida a la protección de la propiedad intelectual; compromisos para la liberalización del mercado de servicios y normas para la mayor transparencia y acceso a las compras públicas. La mayoría de estos temas ha suscitado un importante debate en relación con la necesidad y preparación técnica de los ACP para negociarlos, los potenciales efectos beneficiosos que la UE preconiza y la incertidumbre del impacto de los mismos sobre la pobreza, en especial, en el caso de las economías africanas.

\subsection{Los Acuerdos Mediterráneos y con países de Oriente Medio}

Desde 1995, el marco de las relaciones de la UE con la región mediterránea se engloba en la Asociación Euro-Mediterránea, lanzada en la Conferencia de Barcelona. El denominado «Proceso de Barcelona» dirige las relaciones bilaterales y multilaterales entre ambas regiones. En el marco de la política de la UE con el Mediterráneo, la primera ha celebrado acuerdos con Argelia, Egipto, Israel, Líbano, Jordania, Marruecos, laAutoridad Palestina, Siria y Túnez.

Por otra parte, la UE mantiene relaciones con los países de Oriente Medio situados alrededor del Golfo Pérsico y está negociando la creación de una zona de libre comercio con los seis miembros del Consejo de Cooperación de los Estados Árabes del Golfo (CCG), organización regional que agrupa a Arabia Saudita, Bahrein, los Emiratos Árabes Unidos, Kuwait, Omán y Qatar. La UE está estudiando el modo de fomentar las relaciones económicas bilaterales con Irán en la negociación actual de un acuerdo de comercio y cooperación.

\subsection{Otros acuerdos}

Las relaciones comerciales entre la UE y Turquía, país candidato a la adhesión, continúan en el marco de una Unión Aduanera establecida en 1995 y, según las últimas declaraciones realizadas respecto a la futura adhesión de Turquía, esta no se producirá antes del año 2014. La UE también tiene los ya comentados acuerdos de unión aduanera con Andorra y San Marino, así como un acuerdo de libre comercio con las Islas Feroe.

El primer Acuerdo de Asociación y Cooperación entre la UE y Rusia se firmó en 1997, para un periodo de diez años. Este recoge disposiciones relacionadas con el comercio, la energía, los derechos humanos y la política internacional. Asimismo, la UE ha celebrado acuerdos de asociación y cooperación con otros países de la antiguaUnión Soviética, concretamenteAzerbaiyán, Kazajstán, Kirguizistán, Moldavia, Bielorrusia y Ucrania. Los acuerdos con Moldavia, Rusia y Ucrania se enmarcan en un proceso que podría llevar al establecimiento de una zona de libre comercio entre esos países y la UE.

La UE tiene igualmente establecido un acuerdo de libre comercio con Sudáfrica desde el año 2000, denominado «Trade Development and Cooperation Agreement» (TDCA) y, actualmente, se encuentra, en negociaciones, con Corea del Sur, para el establecimiento de un área de libre comercio futura.

\subsection{Las relaciones comerciales con EEUU, China y Japón}

Paradójicamente, La UE no tiene acuerdos comerciales generales con ninguno de sus principales socios comerciales, esto es, EEUU, China y Japón; el comercio con estos países se canaliza a través de los mecanismos de la OMC. 
La UE ha celebrado diferentes acuerdos para sectores específicos con EEUU y Japón, que dado su limitado carácter sectorial, no se consideran acuerdos comerciales al uso, ni suponen compromisos de creación de áreas de libre comercio, entre otros.

El volumen de comercio con EEUU representa aproximadamente el $25 \%$ de las exportaciones y el $20 \%$ de las importaciones. Las diferencias comerciales que aparecen entre ambos se resuelven ya sea de forma bilateral, o sometiéndolas al órgano de solución de diferencias de la OMC. En el marco de este mecanismo multilateral para resolver los problemas comerciales entre ambos bloques, una tercera parte de los casos denunciados ante la OMC es de EEUU contra la UE y viceversa.

El marco de la OMC también se aplica al comercio entre la UE y China, país que se adhirió a este organismo de comercio mundial en 2001. En la Cumbre UE-China de septiembre de 2006, se estableció el compromiso de iniciar las negociaciones para un Acuerdo de Partenariado y Cooperación, que incluiría aspectos comerciales y de inversión. No existe por el momento ningún trato preferencial comercial, más allá de las rebajas arancelarias que se negocian multilateralmente en el marco de la OMC.

\section{La POLÍTICA COMERCIAL DE LA UE CON AMÉrica LATINA}

\subsection{Los acuerdos ya celebrados: México y Chile}

En el año 2000 entró en vigor el Acuerdo de libre comercio entre la UE y México. Este Acuerdo, firmado en 1997, es el primero entre la UE y un país de América Latina. En virtud de los compromisos del Acuerdo, las exportaciones de la UE percibirán el mismo acceso al mercado mexicano que las de Estados Unidos y Canadá, socios de México en el Tratado de Libre Comercio de América del Norte (TLCAN).

El calendario de liberalización comercial es asimétrico, de forma tal que los plazos liberalizadores establecidos han sido más amplios para México. Así, la UE eliminó todos los derechos de aduana sobre las importaciones de productos industriales de México en 2003 (lo que viene a representar el $94 \%$ de las exportaciones de México a la UE), mientras que México hará lo propio, respecto a las importaciones procedentes de la UE a lo largo de 2007. El Acuerdo recoge, además, compromisos para un trato preferencial en la prestación de servicios y está siendo revisado en ámbitos como la agricultura, los servicios, la inversión y el fortalecimiento del diálogo político.

Las relaciones comerciales entre la UE y Chile se rigen por el Acuerdo de Asociación del año 2002, en vigor desde 2005. El Acuerdo prevé la liberalización progresiva y recíproca del comercio de mercancías a lo largo de un período de diez años y se asienta sobre dos pilares adicionales a la liberalización comercial: el diálogo político y la cooperación al desarrollo (Comisión Europea, 2006b).

En el apartado comercial, el Acuerdo establece el compromiso de liberalizar casi la totalidad de 
los flujos comerciales entre ambos (un 97\%), también en un periodo de diez años. El Acuerdo ha introducido de la misma manera compromisos para la liberalización de las inversiones extranjeras, la apertura recíproca de los mercados de contratación pública y la protección de los derechos de propiedad intelectual.

\subsection{Los Acuerdos en negociación: MERCOSUR y Comunidad Andina (CAN)}

Desde el año 2000 la UE está llevando a cabo negociaciones para liberalizar el comercio con el Mercado Común del Sur (Mercosur), cuyos miembros son Argentina, Brasil, Paraguay, Uruguay y Venezuela. La UE es el socio comercial más importante de los países del Mercosur (representa el 23\%, aproximadamente, de los intercambios comerciales de la región), el primer inversor extranjero en la región y el principal donante de ayuda al desarrollo.

Las negociaciones UE-Mercosur tienen como objetivo sentar las bases para la creación de una zona de libre comercio, mediante la liberalización del comercio de mercancías y servicios y pretende incluir normativas relacionadas con la adjudicación de contratos públicos, los derechos de propiedad intelectual, la política de competencia, las medidas sanitarias y fitosanitarias y normas para la facilitación de inversiones extranjeras, entre otros aspectos. El futuro Acuerdo tiene como objetivo establecer un instrumento para la intensificación de las relaciones políticas, comerciales y económicas entre ambos bloques y contribuir a la reducción de las disparidades socioeconómicas existentes entre ambas regiones (Unión Europea, 2006b).

La UE y la Comunidad Andina (antes Pacto Andino), formada por Colombia, Bolivia, Ecuador y Perú, comenzaron el diálogo político a raíz de la Declaración de Roma en 1996, que, desde entonces, adopta la forma de reuniones periódicas ministeriales y presidenciales. La posibilidad de establecer un Acuerdo de Asociación de base comercial ha ido tomando forma a lo largo de las sucesivas reuniones. Así, en mayo de 2003, se acordó formalmente la negociación de unAcuerdo de Diálogo Político y de Cooperación (suscrito en el 2003)como paso previo a las negociaciones para el Acuerdo de Asociación.

En términos comerciales, la UE es el segundo socio comercial de la CAN (representa el 13\% de los flujos comerciales de la región). Los países CAN se han beneficiado de un acceso preferente (en términos arancelarios) a los mercados europeos desde 1999. En esta fecha, la UE les concedió preferencias unilaterales, en el marco del SPG Drogas, bajo el cual los productos industriales y algunos productos agrícolas han entrado libres de aranceles en los mercados europeos. Este acceso preferencial estaba justificado sobre la base de la responsabilidad compartida en la lucha contra la droga (como zonas productoras por un lado y zonas de consumo, por otro). El Sistema de Preferencias Generalizadas (SPG) fue renovado en el 2005 (tal y como se ha comentado) y se establecieron tres grandes regímenes de aplicación: el régimen general (el menos favorable en términos de concesiones arancelarias), el denominado SPG «plus» o de estímulo del desarrollo sostenible y la Gobernanza y un tercero para los Países Menos Adelantados (PMA) bajo la denominación «todo menos armas». Gracias a estos cambios, los países andinos pasaron a acogerse al SPG+, tras cumplir los criterios de elegibilidad, tales como la ratificación de instrumentos internacionales en materia de derechos humanos, derechos laborales, medio ambiente, drogas, corrupción y la calificación como economía vulnerable. 
El establecimiento del futuro Acuerdo de Asociación UE-CAN vendría a sustituir estas preferencias por un marco recíproco de liberalización comercial. Para la CAN, este Acuerdo proporcionaría a las exportaciones regionales un acceso seguro y estable a los mercados europeos e incrementaría los flujos de inversión en los países miembros. El Acta de Tarija (Bolivia) de 14 junio de 2007, firmada por ambas partes, afirma que el Acuerdo de Asociación deberá ser la expresión de solidaridad entre ambas regiones y tendrá en cuenta de manera apropiada tanto los diferentes niveles de desarrollo económico entre las mismas, como la voluntad, por parte de la UE, de reconocer las asimetrías existentes en la Comunidad Andina.

Así, el Acta establece la posibilidad de otorgar a Bolivia y Ecuador un tratamiento diferenciado (entiéndase más favorable), como economías más vulnerables. Este tratamiento se conoce en el marco de los acuerdos como enfoque de "geometría de variable», donde se plantean calendarios temporales de liberalización y condiciones diferentes en los Acuerdos para unos países frente a otros. Estas concesiones de la UE, parecen encaminadas a incentivar el atractivo del Acuerdo a los dos países más recelosos y más débiles dentro de la CAN, como son Ecuador y, sobre todo, Bolivia.

Teniendo en cuenta las recientes declaraciones de la UE, el Acuerdo de Asociación UE-CAN (así como del futuro Acuerdo con los países centroamericanos) se fundamenta sobre la base de la asociación comercial, política y de cooperación, con las siguientes aspiraciones:

Cuadro 1

Pilares de asociación y aspiraciones del futuro Acuerdo de Asociación UE-CAN

\begin{tabular}{|l|l|}
\hline Asociación económico- comercial: & $\begin{array}{l}\text { Desarrollo del comercio. Atracción de inversiones. } \\
\text { Crecimiento económico. Fomento de la integración } \\
\text { regional. }\end{array}$ \\
\hline Asociación política: & $\begin{array}{l}\text { Promoción de los derechos humanos. Promoción de } \\
\text { la democracia y el buen gobierno. Defensa de estos } \\
\text { valores en la escena internacional. }\end{array}$ \\
\hline Cooperación: & $\begin{array}{l}\text { Consolidación de la estabilidad política, social y } \\
\text { económica.Ayuda para la creación de las condiciones } \\
\text { necesarias para luchar contra la pobreza.Garantía de } \\
\text { un equilibrio adecuado entre los componentes } \\
\text { económicos, sociales y medioambientales en un } \\
\text { contexto de desarrollo sostenible. Mejora de la calidad } \\
\text { de vida de sus poblaciones. }\end{array}$ \\
\hline
\end{tabular}

Fuente: elaboración propia a partir de Comisión Europea (2006d)

El pasado mes de julio, se determinó que las negociaciones para el Acuerdo de Asociación se iniciarían formalmente en septiembre de 2007, en Colombia. Se han establecido catorce mesas de trabajo que reflejan las diferentes vertientes que contendrá el Acuerdo de Asociación: aranceles, agricultura, reglas de origen, asuntos aduaneros y facilitación del comercio, obstáculos técnicos, medidas sanitarias, instrumentos de defensa comercial, servicios, inversión y solución de diferencias, entre otros (Comunidad Andina, nota de prensa, 2007). 


\section{LA RELACIÓN ENTRE LA POLIÍTICA COMERCIAL Y LA COMPETITIVIDAD DE EUROPA}

omo se ha comentado, la política comercial de la UE adquirió especial relevancia como instrumento para mejorar la competitividad de Europa a partir del relanzamiento de la Estrategia de Lisboa de 2005. En la Estrategia de Lisboa de 2000 sólo se contemplaban elementos de carácter interno para la creación de puestos de trabajo y crecimiento económico en la UE. En el 2005, la UE introdujo una acción específica relacionada con el exterior: la necesidad de disponer de mercados abiertos como una de las acciones clave para el logro de los objetivos de Lisboa.

La mejora en la competitividad de Europa se fundamenta, desde ese momento, en dos pilares, interno y externo, estrechamente relacionados. En primer lugar, una dimensión interna para la competitividad: políticas internas adecuadas que den respuesta a los retos competitivos externos y que contribuyan a la apertura de los mercados europeos al comercio y a la inversión procedente del exterior. Adicionalmente, un pilar externo para la competitividad: acciones externas que aseguren reglas de juego claras y justas y un mayor acceso a mercados externos, sobre todo a nuestros futuros principales socios comerciales.

La política comercial comunitaria, adquiere así un papel de relevancia a partir del 2005 y se incorpora como elemento externo a una estrategia que hasta entonces se había configurado fundamentalmente en acciones y políticas de carácter interno.

Desde la perspectiva de la UE, la política comercial juega un papel clave en la mejora de la competitividad, sobre la base de los siguientes ejes esenciales, tal y como se expone a continuación:

Cuadro 2. Política comercial y competitividad de la UE: propuestas de la Comisión Europea (2006)

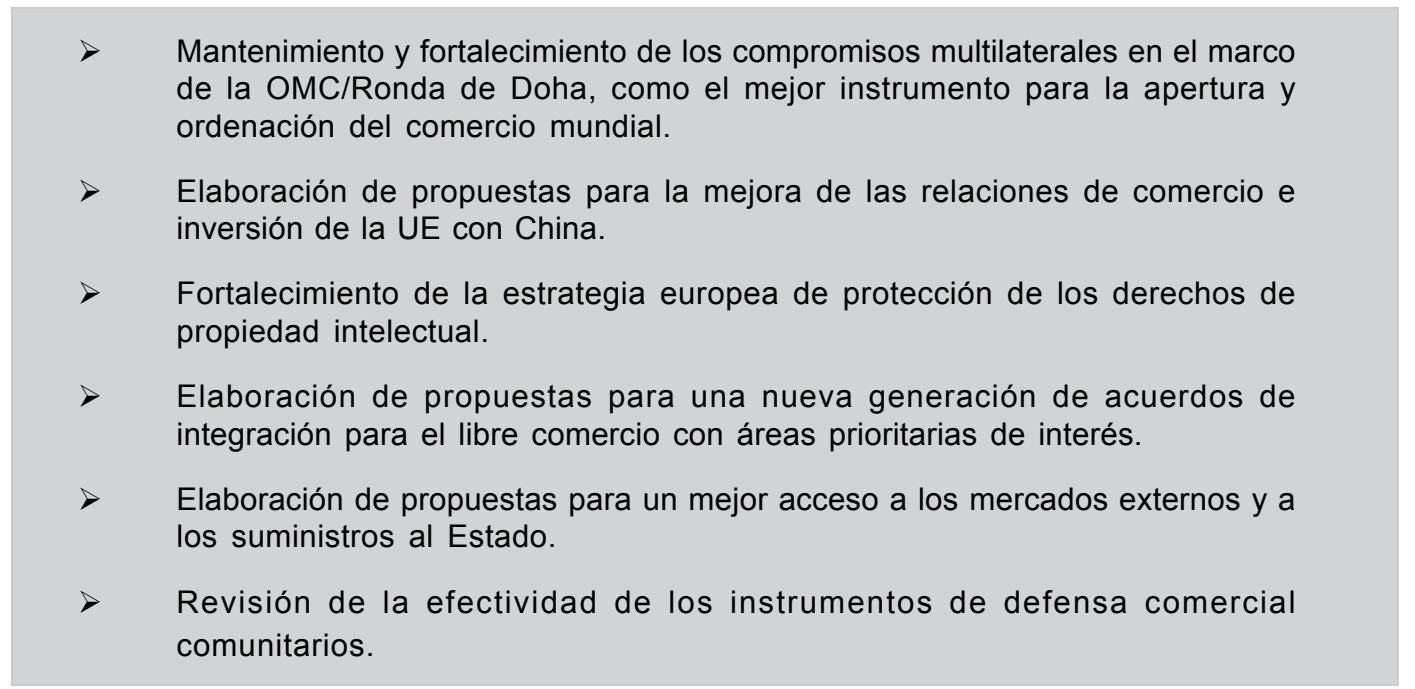

Fuente: elaboración propia a partir de COMISIÓN EUROPEA (2006e): «Global Europe, competing in the World. A contribution to the EU's Growth and Jobs Strategy». Comisión Europea. Bruselas. 
En definitiva y, desde la perspectiva comunitaria, el establecimiento de acuerdos con terceros países, del orden de los descritos, se ha configurado como una herramienta clave para la apertura de mercados. Esta es, desde la perspectiva de la UE, una fuente importante de ganancias de productividad, de crecimiento económico y de generación de empleo.

Desde la óptica de la Comisión, esta apertura refuerza la posición competitiva de Europa en un entorno globalizado donde las economías emergentes ejercen crecientes presiones compe-titivas. Además, la apertura a la que se refiere la Comisión, no tiene que ver sólo con aranceles, sino además, con otro tipo de barreras no arancelarias, como regulaciones o procedimientos. Otras áreas relacionadas con el comercio, como la inversión extranjera, la propiedad intelectual, el comercio de servicios o la contratación pública se han convertido igualmente en piezas fundamentales para la competitividad y acceso de las empresas europeas en el exterior (Comisión Europea, 2006e).

Desde esta perspectiva, es entendible la postura de la UE en cuanto a introducir en los textos de los Acuerdos comerciales con países terceros, disposiciones que incluyen todos estos ámbitos adicionales, referidos a las barreras no arancelarias. La UE considera, asimismo,, que la liberalización en estos ámbitos (servicios, inversión, contratación pública, etc.), es altamente beneficiosa para los países en términos de desarrollo, pues aumenta la competitividad igualmente de los países receptores, al tiempo que reduce los costes del comercio y aumenta la atracción de los países a la inversión extranjera.

\section{Conclusiones}

An la última década, la UE, a través de la Comisión Europea, (con una enorme dedicación institucional), ha realizado grandes esfuerzos retóricos, frente a la necesidad de establecer acuerdos comerciales con terceros países. El establecimiento de estos acuerdos de libre comercio con países terceros, son parte de una estrategia más amplia de búsqueda de una mayor competitividad de Europa. En palabras del comisario europeo de Comercio, Meter Mandelson: «acuerdos bilaterales ambiciosos, con socios cuidadosamente escogidos, pueden crearnuevas oportunidades comerciales, mejorar la competitividad de las egresas de la UE en mercados clave en expansión y allanar el terreno para una futura liberalización, avanzando en ámbitos tales como la inversión, la competencia y l((a contratación pública, donde las normas de la OMC aún no se aplican plenamente» (Mandelson, 2006).

Los nuevos acuerdos de libre comercio han sido clasificados como «globales» por la propia UE, pues establecen, más allá de las estrictas disposiciones comerciales, un marco de relaciones en tres ámbitos: comercio, diálogo político y cooperación. Estos tres pilares se complementan y toman forma bajo los denominados «Acuerdos deAsociación», donde la normativa comercial se mejora con disposiciones que articulan mayores vínculos políticos y medidas de cooperación que se asientan, entre otros elementos, sobre la base de la ayuda al desarrollo de la UE. 
Muchos de estos acuerdos se están estableciendo con áreas regionales (MERCOSUR, CAN, CARIFORUM, ASEAN, SADC, ECOWAS en África, entre otros) y no con países individuales. La UE afirma que esta dinámica propicia que los propios Acuerdos potencien la integración regional. Además, otros objetivos, como la lucha contra la pobreza, la cohesión social, la atracción de inversiones, la mayor transparencia en la contratación pública, la defensa de los derechos humanos, la protección del medio ambiente, entre otros aspectos, han sido señalados como ambiciosos objetivos a perseguir por estos acuerdos.

La UE defiende así un modelo de relaciones exteriores, donde se pretende incluir una serie de valores, además de garantizar un mejor acceso de las empresas europeas a los mercados externos, en línea con los objetivos de Lisboa de lograr una mayor competitividad para Europa, mediante el crecimiento económico y la creación de puestos de trabajo.

La consecución de todos estos objetivos económicos, políticos y humanos, que es desde luego muy deseable desde el punto de vista de coherencia de políticas de la UE, plantea no pocas dificultades. No son pocos los que afirman que los intereses estratégicos de mejorar el acceso de las empresas europeras a los mercados exteriores, están siendo priorizados por encima de otros más complejos de conseguir en el ámbito de los derechos sociales y del desarrollo humano; por esta razón, no es fácil que los objetivos sean compatibles.

Se plantean así numerosos interrogantes que tienen que ver con los compromisos que incluyen la mayor parte de los Acuerdos de la UE con terceros países: ¿cómo asegurar que la liberalización comercial no perjudica a sectores y colectivos más vulnerables en los países?; ¿cómo conseguir que la liberalización de las inversiones incremente las inversiones a los sectores y países que más lo necesiten en términos de desarrollo y no sólo a los más rentables?; ¿en qué medida la liberalización de los servicios no contribuirá a la desaparición de las empresas locales?; ¿beneficiarán por igual las medidas de protección intelectual a unos y a otros?; ¿cómo se pueden garantizar acuerdos de beneficios equilibrados a través de negociaciones entre socios generalmente tan dispares?...por nombrar algunas cuestiones.

Conviene recordar que la UE no es ni en el ámbito político ni en el económico una sola entidad, sino que está formada por 27 Estados diferentes, sobre los que las diferentes instituciones prevalecen sólo en algunos de ellos. Aunque la política comercial es, como se ha comentado, competencia exclusiva de la UE, es lógico pensar que sobre la misma, se ejercen numerosas presiones institucionales, sociales e, igualmente, empresariales.

La UE se está configurando de forma creciente como un actor político y económico de primer orden. Si en el marco de su política comercial se defiende un modelo de acuerdos globales, de «nueva generación», muy ambicioso en contenidos y objetivos, con más valores que los de otros actores internacionales (como EEUU o China) y sobre todo, coherentes con la política de desarrollo, la legitimidad futura de la UE estará condicionada a que los Acuerdos resulten finalmente en beneficios equilibrados para todos y no sólo para Europa y sus ciudadanos. El reto está servido. 


\section{BibLiografía}

COMISIÓN EUROPEA (2006a): Instrumentos de Defensa Comercial Europeos en una Economía Global en Transformación. Libro Verde para Consulta Pública. Comisión de las Comunidades Europeas. Bruselas.

COMISIÓN EUROPEA (2006b): La Unión Europea, América Latina y el Caribe: una Asociación Estratégica. Dirección General de Relaciones Exteriores. Comisión Europea. Bruselas.

COMISIÓN EUROPEA (2006c): The European Consensus on Development. Dirección General de Desarrollo, Comisión Europea. Bruselas.

COMISIÓN EUROPEA (2006d): Directrices para la Negociación de os Acuerdos de Asociación con Centroamérica y la Comunidad Andina. Bruselas.

COMISIÓN EUROPEA (2006e): Global Europe, competing in the World. A contribution to the EU's Growth and Jobs Strategy. Comisión Europea. Bruselas.

COMISIÓN EUROPEA(2006f): Overview of Bilateral Negotiations involving TradeAgreements. Updated on 04/12/2006. Comisión de las Comunidades Europeas. Bruselas.

COMISIÓN EUROPEA (2004): El Sistema de Preferencias Generalizadas de la Unión Europea. Comisión de las Comunidades Europeas. Bruselas.

COMISIÓN EUROPEA (2002): La Globalización en Beneficio de Todos. La Unión Europea y el Comercio Mundial. Comisión de las Comunidades Europeas. Bruselas.

COMUNIDAD ANDINA (2007). Nota de prensa del 17 de julio de 2007. Secretaría General de la Comunidad Andina.

GONZÁLEZ RABANAL, M. de la C. (2006): en LINDE, E. Políticas de la Unión Europea. Colex. Madrid.

ORGANIZACIÓN MUNDIAL DEL COMERCIO. (2007): Examen de las Políticas Comerciales: Comunidades Europeas. Secretaría de la OMC. Ginebra.

MANDELSON, P. (2006): Acuerdos Bilaterales en la Política Comercial de la Unión Europea. Discurso del Comisario Europeo de Comercio. London School of Economics. 9 de octubre.

MANDELSON,P. (2007): Política Comercial de la Unión Europea después de Hong Kong. Discurso del Comisario Europeo de Comercio en Berlín, 23 de enero.

REAL INSTITUTO ELCANO (2005): The Euro-Mediterranean Partnership: Assesing the First Decade. Real Instituto Elcano de Estudios Internacionales y Estratégicos. Madrid.

WEBS DE REFERENCIA

Comisión Europea. Dirección General de Comercio: Http://ec.europa.eu/trade/index_en.htm Parlamento Europeo. Comisión del Comercio Internacional. Http://www.europarl.europa.eu/ committees/inta_home_en.htm

Consejo de la Unión Europea. Asuntos generales y relaciones exteriores.

Http://www.consilium.europa.eu/ 
